\title{
Search of Dark Matter with space experiments
}

\author{
Aldo Morselli* \\ INFN Roma II and University of Roma "Tor Vergata" \\ E-mail: ala do.morseliêroma2.infn. itt
}

Abstract: The energy domain between $10 \mathrm{MeV}$ and hundreds of $\mathrm{GeV}$ is an essential one for the multifrequency study of extreme astrophysical sources and the experiment GLAST will fill this energy region from the fall of 2006. GLAST is particularly interesting for the supersymmetric particle search because, if neutralinos make up the dark matter of our galaxy, the detection of gamma rays due to pair annihilation of dark matter particles in the Milky Way halo is a viable techniques to search for supersymmetric dark matter candidates. Here we will present the achievable limits with this experiment.

Experimental cosmology has been steadily progressing over the last few years. The emerging picture [i] i i is a cosmological average matter density of $\Omega_{m}=0.27 \pm 0.04$ which is much larger than the baryon density $\Omega_{b}=0.044 \pm 0.004$.

The favored candidate for the nonbaryonic component is a neutral weakly-interacting massive particle (WIMPs) with a mass in the range from tens of $\mathrm{GeV}$ to $\mathrm{TeV}$. The most popular case is that of the lightest neutralino in $R$-parity conserving supersymmetric models. Considerable effort has been put into the search for dark matter WIMPs in the last decade, with several complementary techniques applied (for a recent review, see, e.g. [2i]). One route worth being explored is provided by indirect signatures. Neutralinos should pervade the Milky Way halo and be concentrated at the galactic centre and in the cores of the sun and earth. As they mutually annihilate, they should produce high energy photons and antimatter cosmic-rays and should therefore generate spectral distortions in the corresponding backgrounds. Since WIMPs are bound to the Galaxy, they have non-relativistic velocities; hence the neutralino annihilation into two $\gamma$ 's and a $\gamma$ and a $\mathrm{Z}$ as final states can give rise to $\gamma$-rays with unique energies $E_{\gamma}=M_{\chi}$ and $E_{\gamma}^{\prime}=M_{\chi}\left(1-m_{z}^{2} / 4 M_{\chi}^{2}\right)$. All the other annihilation processes will also give $\pi^{0}$ 's that will decay in a continuum gamma-ray flux. The key problem is to separate the two different (standard and exotic) contributions. As most photons are produced in the hadronization and decay of $\pi^{0} \mathrm{~s}$, the shape of the photon spectrum is always peaked at half the mass of the pion, about $70 \mathrm{MeV}$, and it is symmetric around it on a logarithmic scale (sometimes this feature is called the " $\pi^{0}$ bump").

\footnotetext{
${ }^{*}$ Speaker.
} 

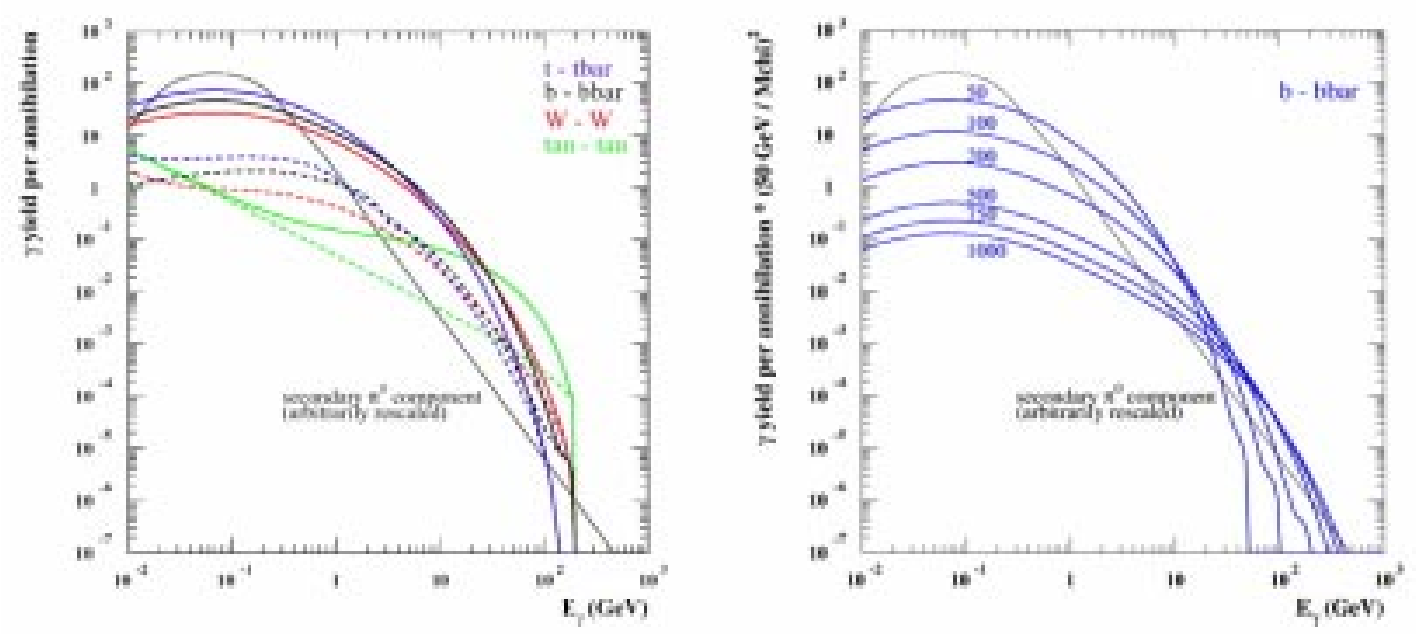

Figure 1: In the left panel: differential yield per annihilation for a few sample annihilation channels and a fixed WIMP mass $(200 \mathrm{GeV})$. The solid lines are the total yields, while the dashed lines are components not due to $\pi^{0}$ decays. For comparison the emissivity, with normalization arbitrarly rescaled, from the interaction of primaries with the interstellar medium is shown. In the right panel: differential yields per annihilations for a fixed annihilation channel $(b \bar{b})$ and for a few sample values of WIMP mass, rescaled with the inverse the WIMP mass squared.

The signal arises in processes which all have the same energy scale, i.e. $2 M_{\chi}$, therefore, the WIMP induced flux is spectral index free and shows a sharp cutoff when $E_{\gamma}$ approaches the WIMP mass. Conversely the background spectrum above an energy of about $1 \mathrm{GeV}$ can be described by a power law of the form $\phi_{\text {back }} \propto E_{\gamma}^{-\alpha}$ with the same spectral index as the dominant primary component, i.e. the proton spectral index $\alpha=2.72$. This is shown in the right panel of figure ind where we plot the differential photon yield per annihilation times the inverse of WIMP mass squared, for a few values of the WIMP mass, and assuming WIMPs have a single dominant decay channel ( $b \bar{b}$ in the case displayed). In the same figure, for comparison, the spectral shape of the background is shown: as it can be clearly seen, one may hope to identify the WIMP induced component as a distortion of the background spectrum at relatively high energies. For a given WIMP mass, the photon yields in the different annihilation channels are analogous, as shown in the left panel of figure 11]: solid curves indicate the total photon yield, while dashed curves indicate the photon yield in radiative processes, i.e. in all processes rather than $\pi^{0}$ decays. The spectrum for the $t \bar{t}$ and $W^{+} W^{-}$channels are very close to one for $b \bar{b}$ (differences are mainly given by prompt decays before hadronization); only in the $\tau^{+} \tau^{-}$case, radiative photon emission is dominant, still with a large bump due to the hadronic decay modes of $\tau$ leptons $[\underline{\bar{\beta}}]$. Figure $\underline{2}_{1}^{\overline{2}}$ shows the EGRET data from the Galactic center together with the diffuse gamma ray background flux expected from the standard interactions and propagation models of cosmic ray protons and electrons and an example of the flux due to neutralino annihilation in the dark matter halo [3in] . In this case the signal is for a $\sim 80 \mathrm{GeV}$ neutralino and for the $W^{-} W^{+}$annihilation channel (the spectral shape of the other channels is very similar). 


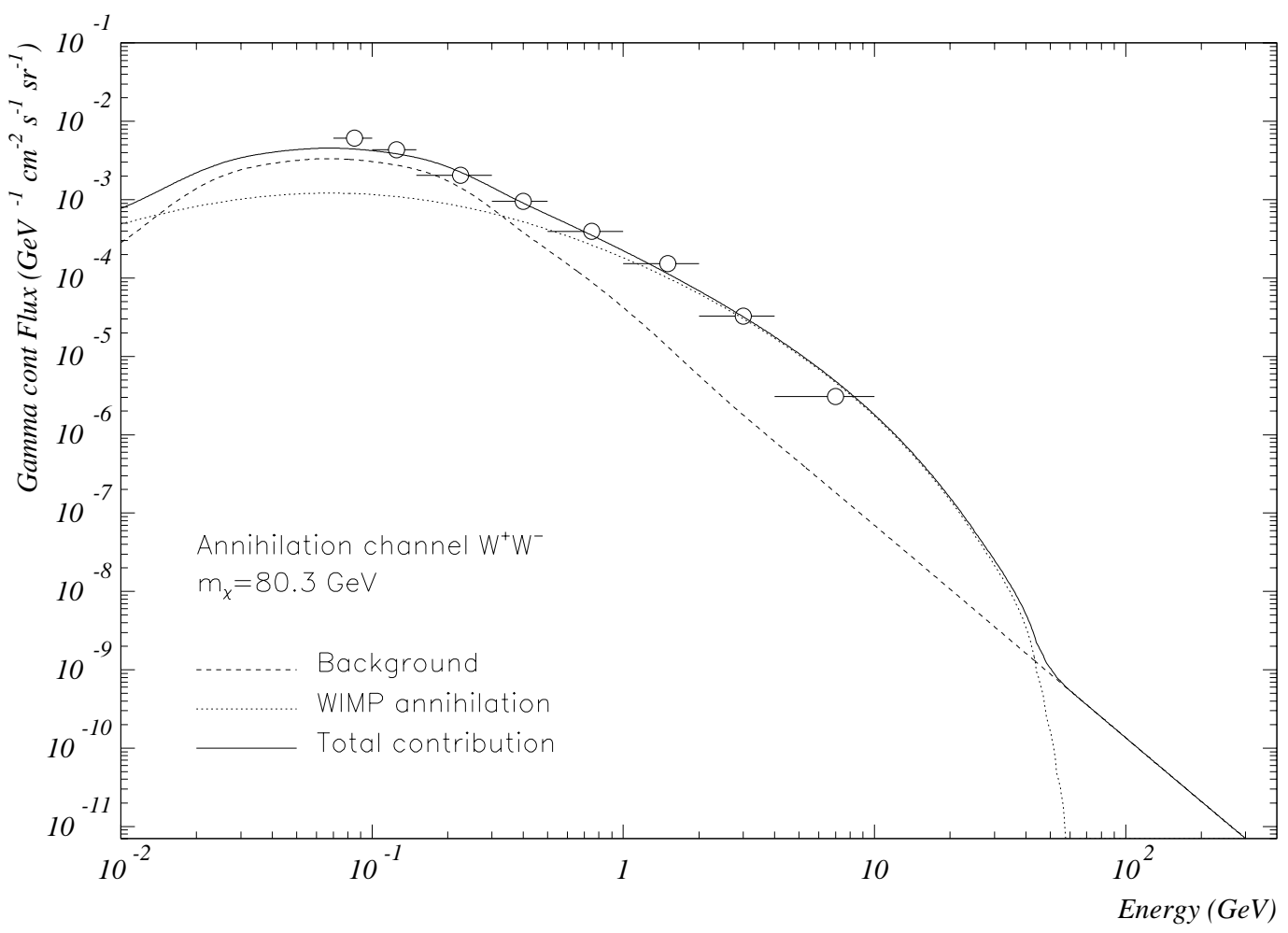

Figure 2: Fit of the EGRET Galactic Center $\gamma$-ray data for a sample WIMP models. with $M_{\chi}=80.3 \mathrm{GeV}$ and $W^{-} W^{+}$annihilation channel.

\section{GLAST}

GLAST [i5i] is a next generation high-energy gamma-ray observatory designed for making observations of celestial gamma-ray sources in the energy band extending from $20 \mathrm{MeV}$ to more than $300 \mathrm{GeV}$. The principal instrument of the GLAST mission is the Large Area Telescope (LAT) that is being developed jointly by NASA and the US Dept. of Energy (DOE) as a mission involving an international collaboration of particle physics and astrophysics communities from 26 institutions in the United States, Italy, Japan, France and Germany. The main scientific objects are the study of all gamma ray sources such as blazars, gamma-ray bursts, supernova remnants, pulsars, diffuse radiation, and unidentified high-energy sources. Many years of refinement has led to the configuration of the apparatus shown in figure formed by: • Si-strip Tracker Detectors and converters arranged in 18 XY tracking planes for the measurement of the photon direction. • Segmented array of CsI(TI) crystals for the measurement the photon energy. • Segmented Anticoincidence Detector (ACD).

The pair-converted positron and electron of the gamma, are tracked through the silicon and tungsten of the tracker with the goal of find the best one or two trajectories, depending on the incident energy. Multiple scattering is key to this analysis, in that it is the dominant 


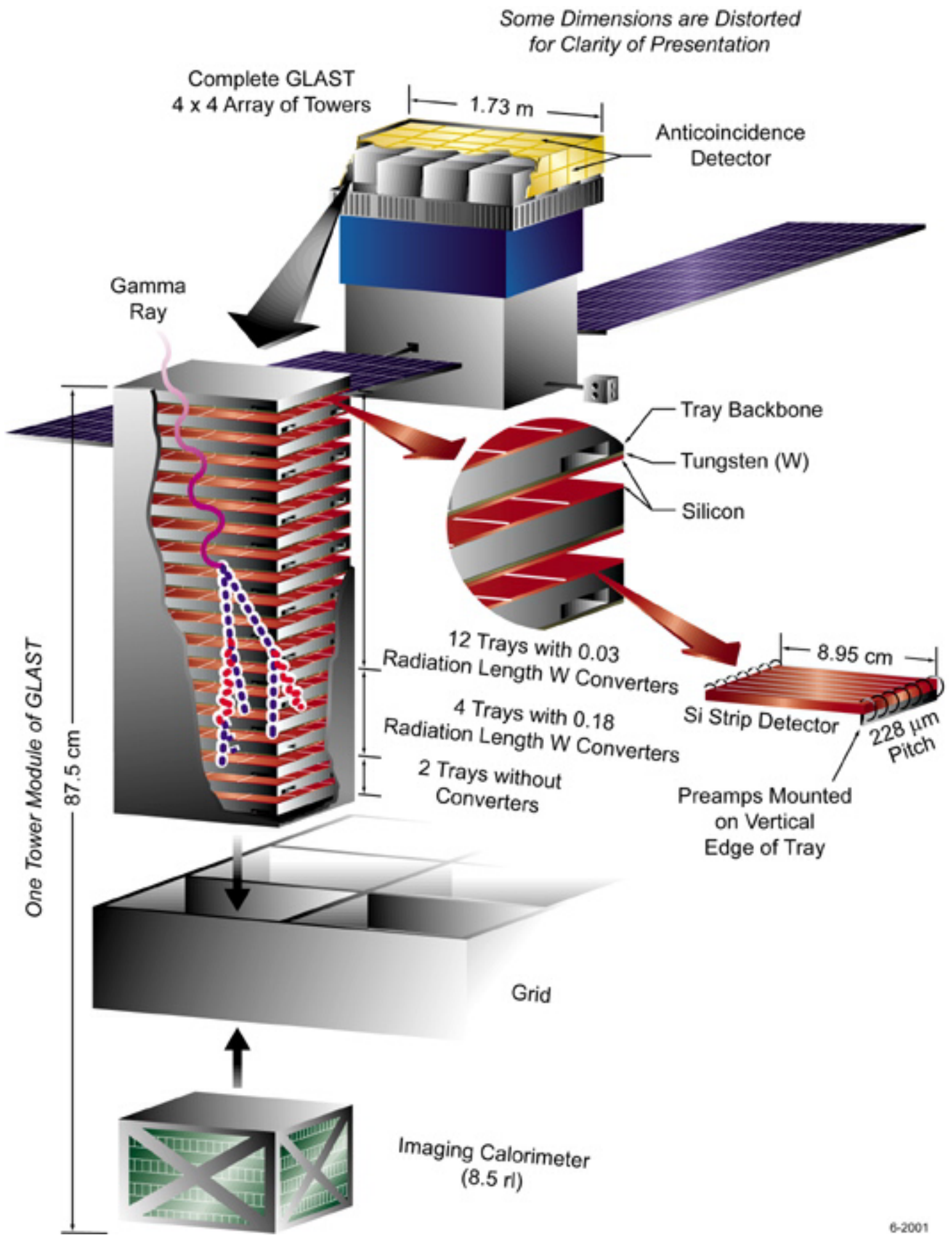

Figure 3: The GLAST instrument.

error contribution below a few GeV. A Kalman filter technique is used in the tracker to account for this effect. It basically follows trajectories, accounting for energy-dependent error introduced by material in the tracker and predicting a cone in which to look for hits to associate in the next layer. At present, energy in the calorimeter is lumped together into 

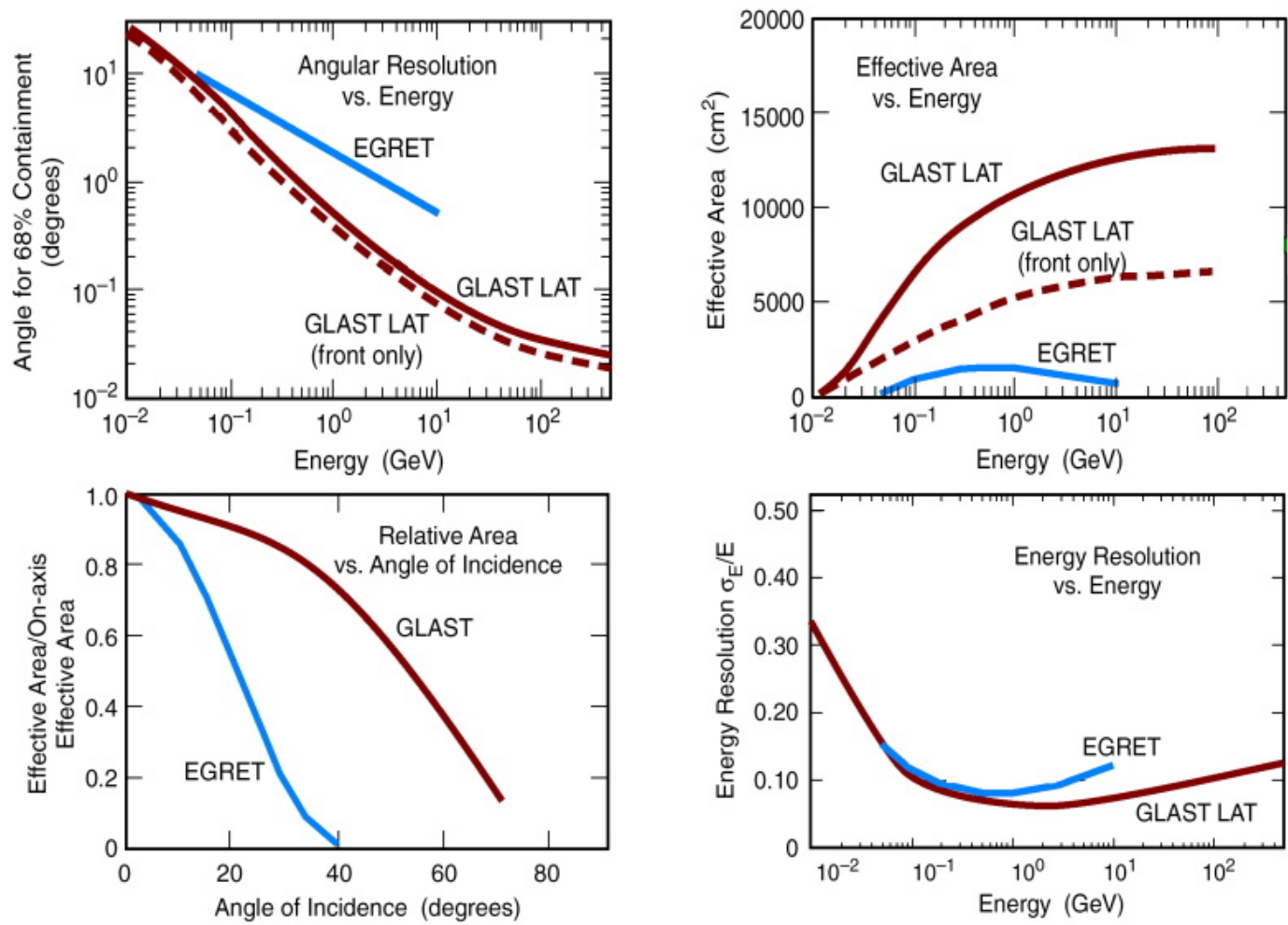

Figure 4: Instrument performance, including all background and track quality cuts.

a single cluster from whose moments are derived the energy and direction. The energy is used to seed the track finding, since the multiple scattering errors are energy dependent. The calorimeter is not thick enough to contain all the shower energy above few GeV. Corrections are required to estimate the leakage energy. This has been done in two ways: correlating the total energy with the energy measured in the last layer; and by fitting the expected shape of the shower against a standard function. Tracks are extrapolated back to the anti-coincidence device to help distinguish background events. The data, filtered by onboard software triggers, are streamed to the spacecraft for data storage and subsequent transmittal to groundbased analysis centers. The Tracker provides the principal trigger for the LAT, converts the gamma rays into electron-positron pairs, and measures the direction of the incident gamma ray from the charged-particle tracks. It is crucial in the first levels of background rejection for providing track information to extrapolate cosmic-ray tracks to the ACD scintillator tiles, and it is important for further levels of background analysis due to its capability to provide highly detailed track patterns in each event. The main characteristics of the detector, extensively studied with Monte Carlo and beam tests, are shown in figure $4 \overline{4}$. They are an energy range between $20 \mathrm{MeV}$ and $300 \mathrm{GeV}$, a field of view of $\sim 3 \mathrm{sr}$, an energy resolution of $\sim 5 \%$ at $1 \mathrm{GeV}$, a point source sensitivity of $2 \times 10^{-9}$ $\left(\mathrm{ph} \mathrm{cm}^{-2} \mathrm{~s}^{-1}\right.$ ) at $0.1 \mathrm{GeV}$, an event deadtime of $20 \mu \mathrm{s}$ and a peak effective area of 10000 $\mathrm{cm}^{2}$, for a required power of $600 \mathrm{~W}$ and a payload weight of $3000 \mathrm{Kg}$. A more detailed description of the main GLAST parameters can be found in 


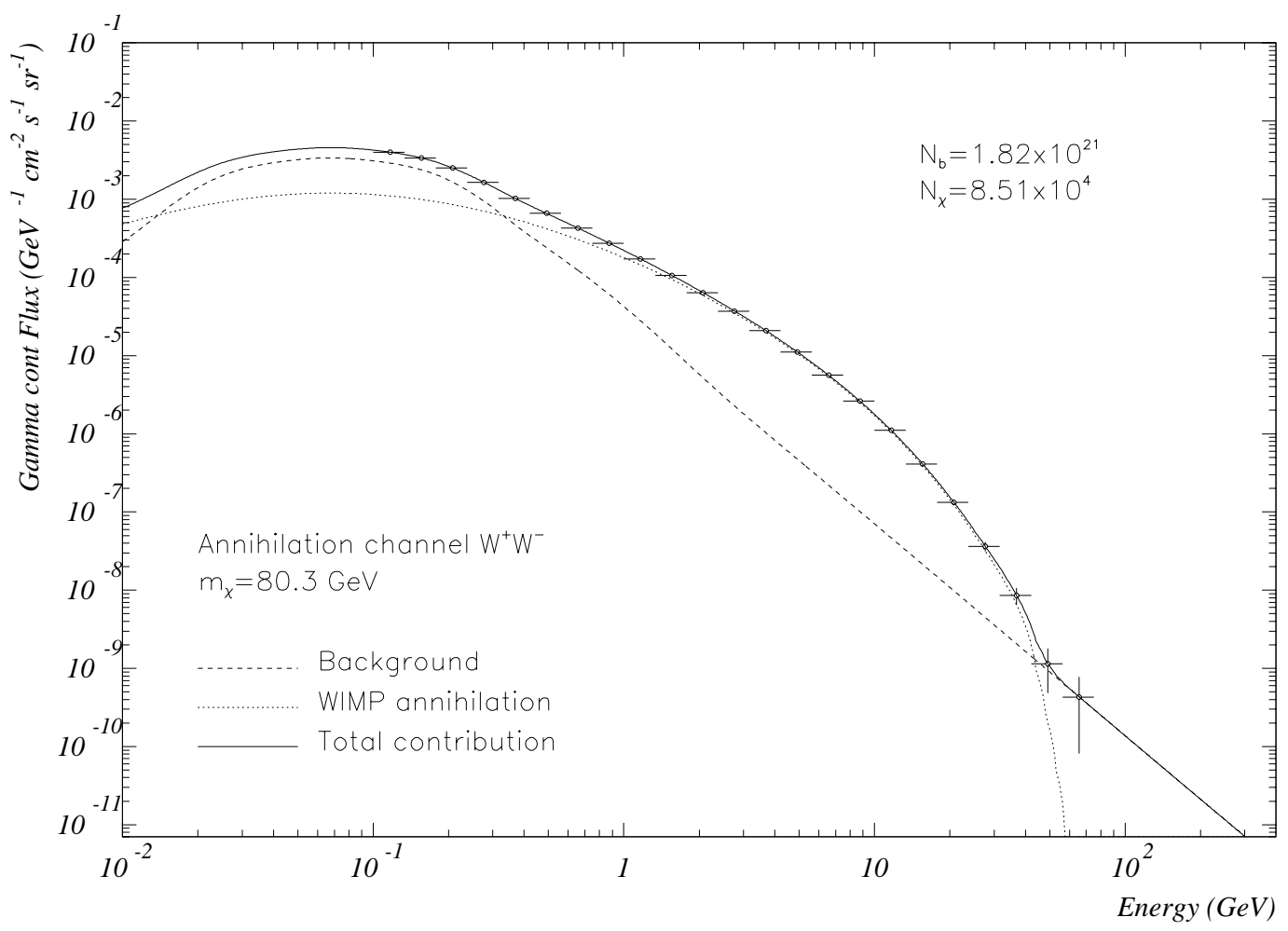

Figure 5: Same fluxes of figure with the kind of statistical errors that it is expected in three years with GLAST.

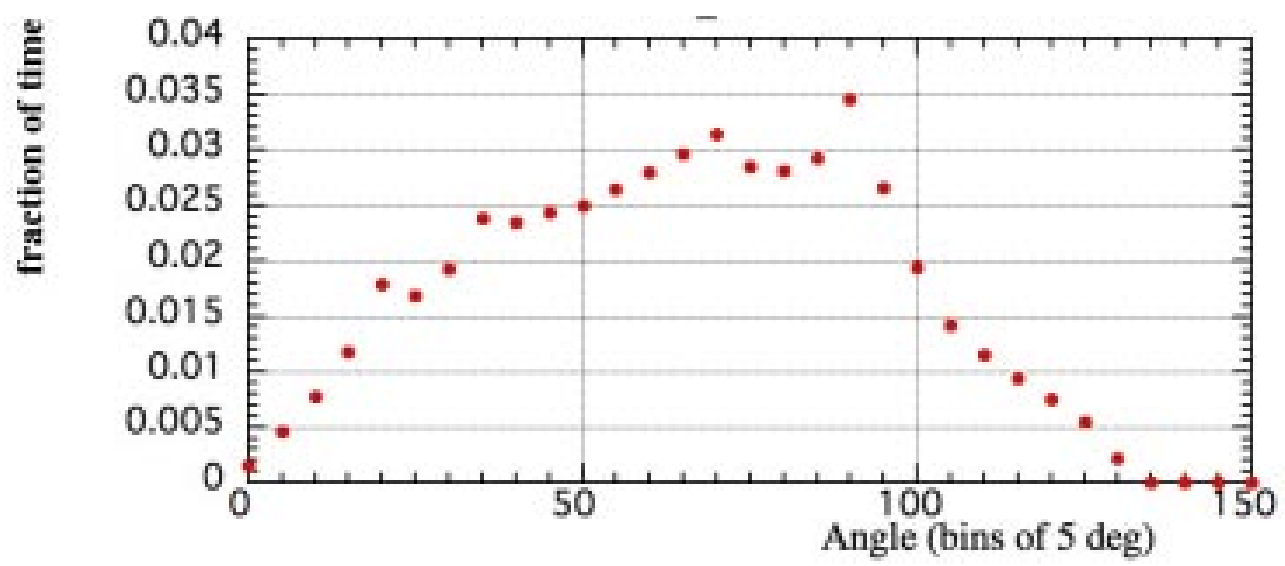

Figure 6: Distribution of observing time with inclination angle for the declination of the Galactic center

Figure expected in two years with GLAST [i⿱㇒冋]| $]$. It can be seen that GLAST will have the necessary statistical, angular and energetic accuracy to distinguish the two kind of spectral shape. Because GLAST will point always at the zenith, to calculate the statistical errors of figure 


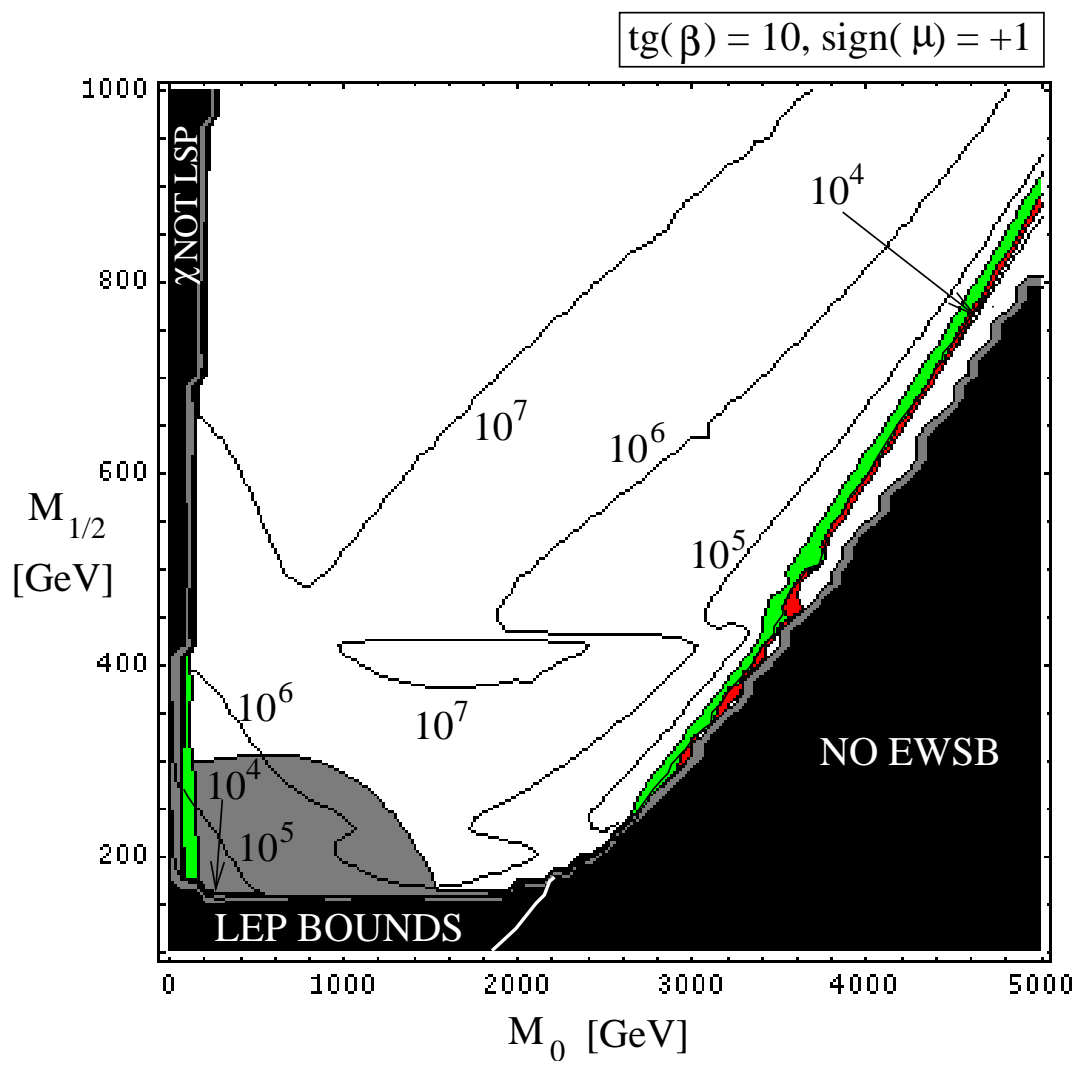

Figure 7: Contour plot in the mSUGRA $\left(m_{0}, m_{1 / 2}\right)$ plane, for the value of the normalization factor $N_{\chi}$, that allows the detection of the neutralino $\gamma$ ray signal with GLAST. In the green region $0.13 \leq \Omega_{\chi} h^{2} \leq 1$, while the red region corresponds to the WMAP range $0.09 \leq \Omega_{\chi} h^{2} \leq 0.13$. The black region corresponds to models that are excluded either by incorrect EWSB, LEP bounds violations or because the neutralino is not the LSP. In the dark shaded region $m_{h_{0}}<114.3 \mathrm{GeV}$ and $h_{0}$ is the lightest Higgs.

'5י, we derive the fraction of time the GC center is visible by the instrument. We simulate a sky survey with $\pm 35^{\circ}$ rocking and take into account the loss of exposure due to the South Atlantic Anomaly (SAA) passages, which corresponds to about $14.2 \%$ of the orbiting time. The target direction was considered to be viewable if its zenith angle was no more than 105 deg. We find that the fraction of time that the GC is within the GLAST field of view is equal to 0.592 , and that the net fraction of time that the source can be observed is 0.508 ; the reduction in effective area for sources which are not located at the instrument zenith gives a mean effective area equal to $60 \%$ of the peak effective area. The distribution of observing time with inclination angle for the declination of the Galactic center is shown in figure ${ }_{-i}^{i} \overline{-}_{i}^{i}$ The figure divides this fraction into inclination angle ranges and the sum of all values is 1 .

We focus now on the most widely studied WIMP dark matter candidate, the lightest neutralino, in the most restrictive supersymmetric extension of the Standard Model, the minimal supergravity (mSUGRA) framework [īin]

After fixing the five mSUGRA parameters at the unification scale, we extract from 


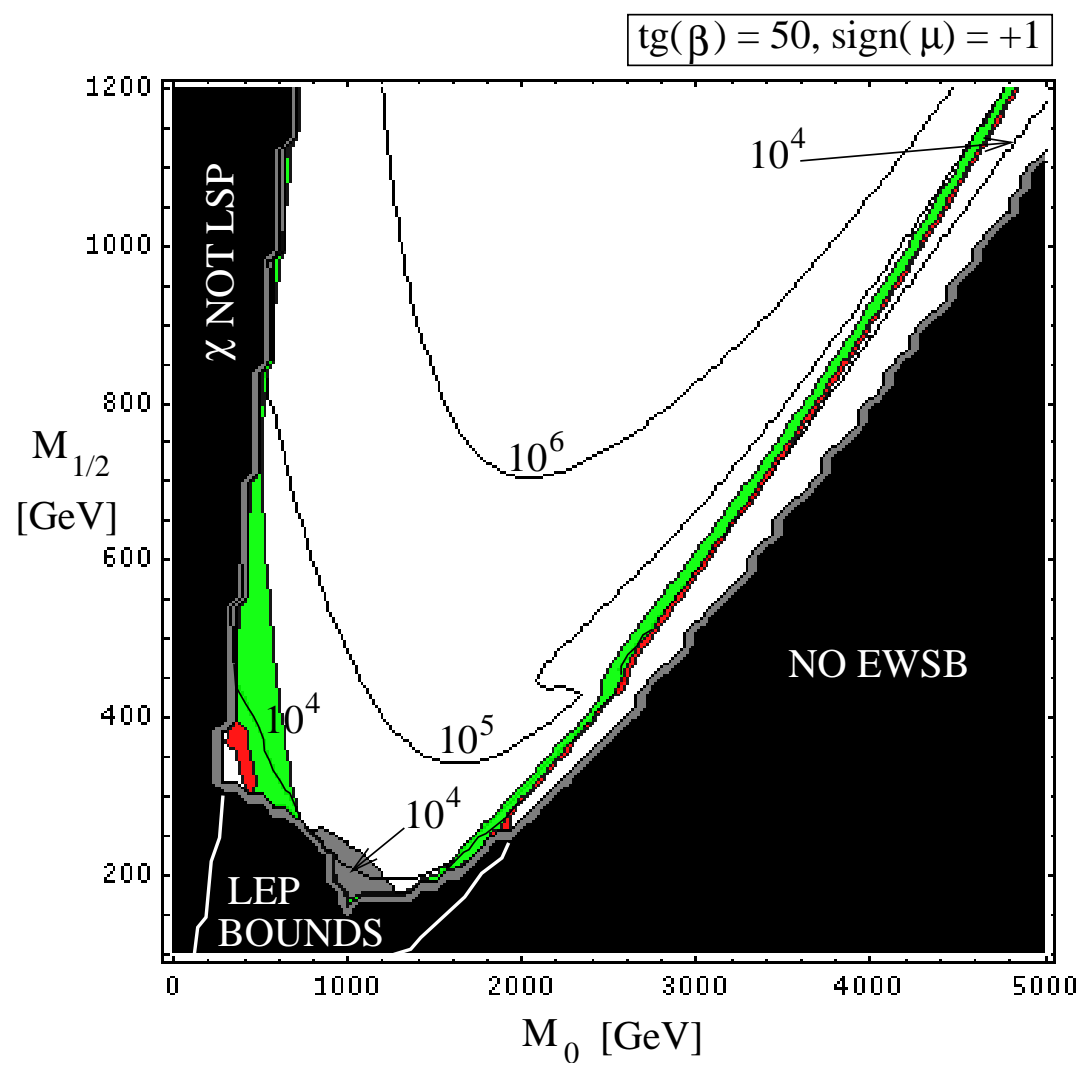

Figure 8: Same as figure $\overline{1}_{1}^{1}$ with $\tan \beta=50$

the ISASUGRA output the weak-scale supersymmetric mass spectrum and the relative mixings. Cases in which the lightest neutralino is not the lightest supersymmetric particle or there is no radiative EWSB are disregarded. The ISASUGRA output is then used as

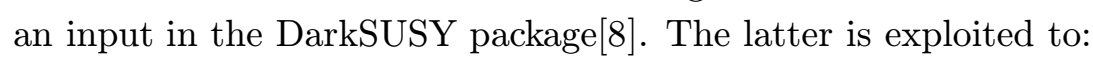

- reject models which violate limits recommended by the Particle Data Group 2002 (PDG) $[\overline{9}]$;

- compute the neutralino relic abundance, with full numerical solution of the density evolution equation including resonances, threshold effects and all possible coannihi-

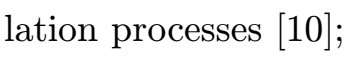

- compute the neutralino annihilation rate at zero temperature in all kinematically allowed tree-level final states (including fermions, gauge bosons and Higgs bosons;

- estimate the induced gamma-ray yield by linking to the results of the simulations performed with the Lund Monte Carlo program Pythia [i] 1 in as implemented in the DarkSUSYpackage.

Fixing $\tan \beta, A_{0}$ and $\operatorname{sgn}(\mu)$, we have performed a scan in the $\left(m_{0}, m_{1 / 2}\right)$ plane searching for the minimum dark matter density, in the GC region, needed to be able to single out the neutralino annihilation signal with GLAST. First we estimate the statistical error $(1 \sigma)$ 


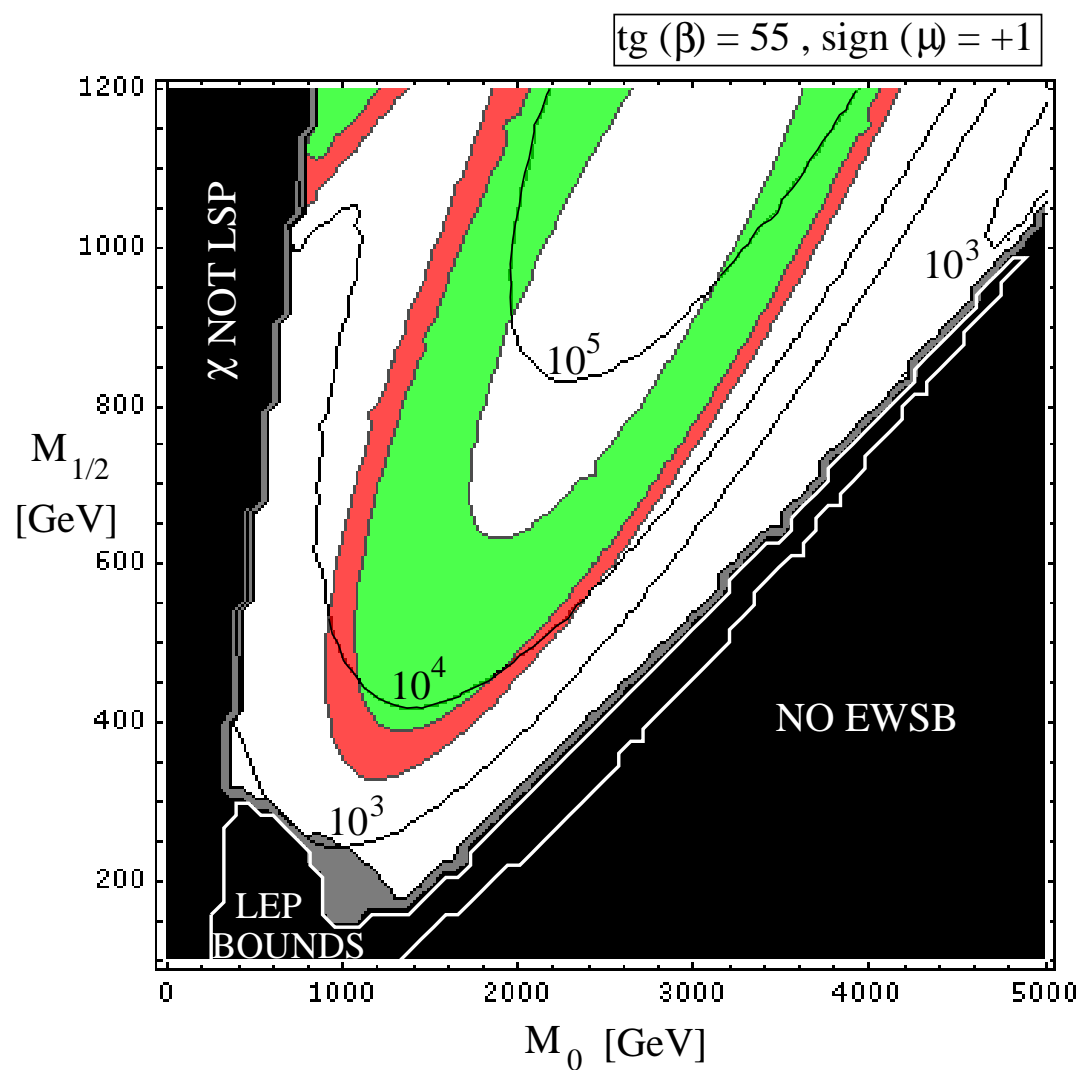

Figure 9: Same as figure $\overline{7}$ in with $\tan \beta=55$

on GLAST data to be the square root of the number of events. To compute the latter we multiply the flux by the effective area of the detector, by the total observational time and the angular resolution $\Delta \Omega=10^{-5} \mathrm{sr}$. Then for each value of the pair $\left(m_{0}, m_{1 / 2}\right)$ we compute the difference between the fluxes $\phi_{\gamma}=\phi_{b}+\phi_{\chi}=N_{b} S_{b}+N_{\chi} S_{\chi}$ and $\phi_{\gamma}^{\prime}=\phi_{b}=N_{b} S_{b}$. If $\phi_{\gamma}-\phi_{\gamma}^{\prime}>3 \sigma$ we consider the SUSY model with those values of $\left(m_{0}, m_{1 / 2}\right)$ to be detectable by GLAST.

Figures ' years the supersymmetric dark matter hypothesis [1] $\left.12_{1}^{n}\right]$. The figure shows in the $\left(m_{0}, m_{\frac{1}{2}}\right)$ plane, the iso-contour regions for the minimum allowed value of the neutralino density in a $\Delta \Omega=10^{-5} s r$ region around the galactic center. The density depends on the halo shape of the neutralino distribution, that is still matter of debate and can vary from a value of $N_{\chi}=3 \times 10^{1}$ for an isotermal profile up to $N_{\chi}=10^{4}$ for a NFW profile [1.31] and up to $N_{\chi}=10^{7}$ for a Moore profile [1] ind $_{1}$. GLAST indeed can explore a good portion of the supersymmetric parameter space especially at large values of $\tan \beta$ and if the halo has a NFW (or steeper) profile. This is a very steep $(1 / r)$ profile but consistent with available dynamical constraints on the Galaxy.

This effort will be complementary to a similar search for neutralinos looking with

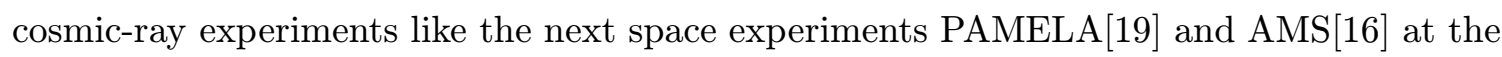

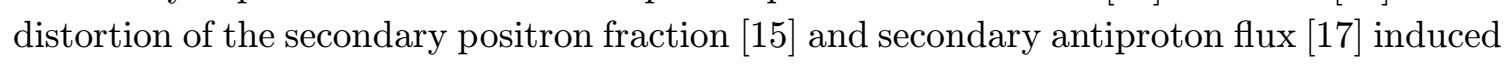



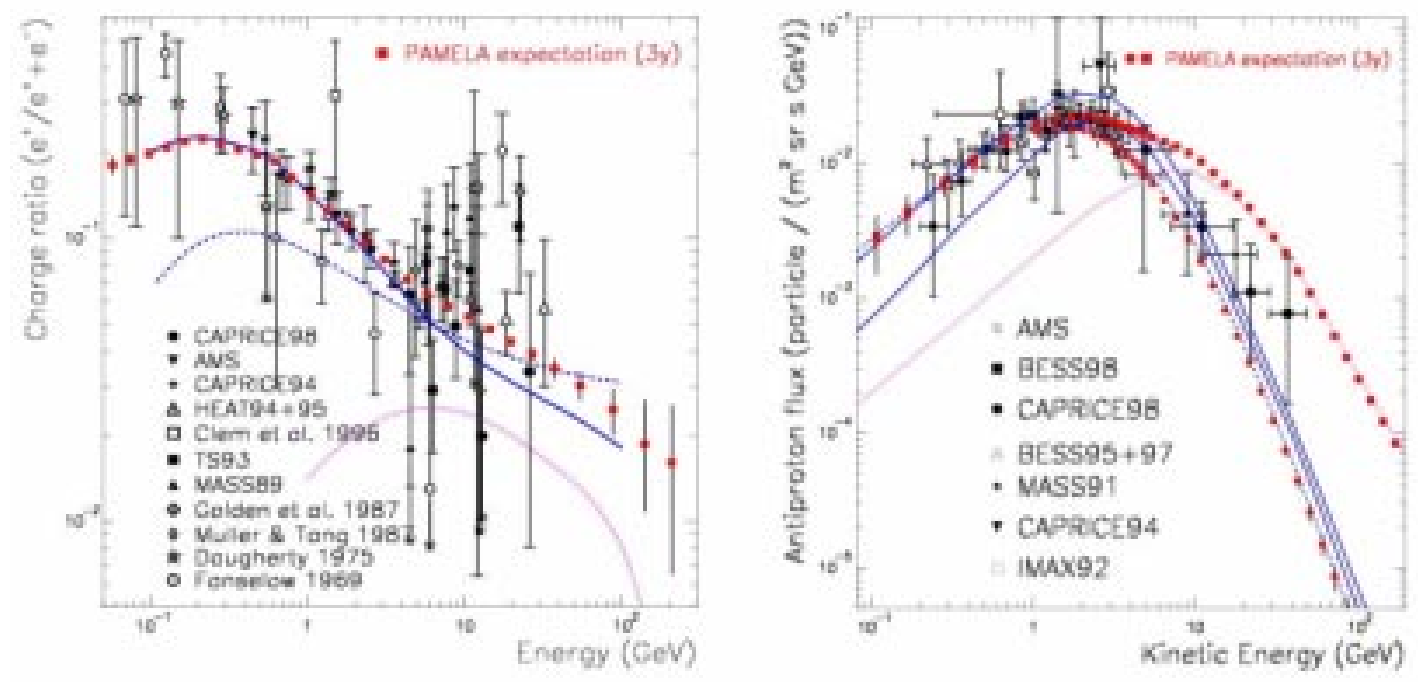

Figure 10: Distortion of the secondary positron fraction (on the left) and secondary antiproton flux (on the right) induced by a signal from a heavy neutralino. The PAMELA expectations in the case of exotic contributions are shown by red squares. For the experimental results and standard theoretical predictions see [i] $\left.{ }^{\prime} \bar{\eta}\right]$

by a signal from a heavy neutralino. As an example, the expected data from the experiment PAMELA in the annihilation scenario for three years of operation are shown by squares in figure $1 \overline{1} \underline{0}$ for both the positron and antiproton fluxes.

This effort will be also complementary to the ground gamma-ray experiments. In figure 11 1 ' (top) the sensitivities of present and future detectors in the gamma-ray astrophysics is shown. The predicted sensitivity of a number of operational and proposed Cherenkov telescopes, CELESTE, STACEE, VERITAS, Whipple is for a 50 hour exposure on a single source. EGRET, GLAST, MILAGRO, ARGO, AMS and AGILE sensitivity is shown for one year of all sky survey. For AMS only the estimate for two points exist [1] $1 \overline{8} \overline{-1}$. The first, at $1 \mathrm{GeV}$, is for the AMS-conversion mode and the second, at 10 $\mathrm{GeV}$, is for the calorimeter mode (see [i] $2 \cdot 10^{-5}$ photons $\mathrm{cm}^{-2} \mathrm{~s}^{-1} \mathrm{sr}^{-1}(100 \mathrm{MeV} / \mathrm{E})^{1.1}$, typical of the background seen by EGRET at high galactic latitudes. The source differential photon number spectrum is assumed to have a power law index of -2, typical of many of the sources observed by EGRET and the sensitivity is based on the requirement that the number of source photons detected is at least 5 sigma above the background. Note that on ground only MILAGRO and ARGO will observe more than one source simultaneously. The Home Pages of the various instruments are at http://www-hfm.mpi-hd.mpg.de/CosmicRay/CosmicRaySites.html. If the neutralino mass is greather then $300 \mathrm{GeV}$, ground experiments will have a better opportunity to discover any signals [2] $[20-1]$.

In figure 1'i is (bottom) it is shown the time of operation and energy range of space X-ray satellite and gamma-ray experiments . Note that AGILE and GLAST will cover an interval not covered by any other experiments. Note also the number of other experiments 

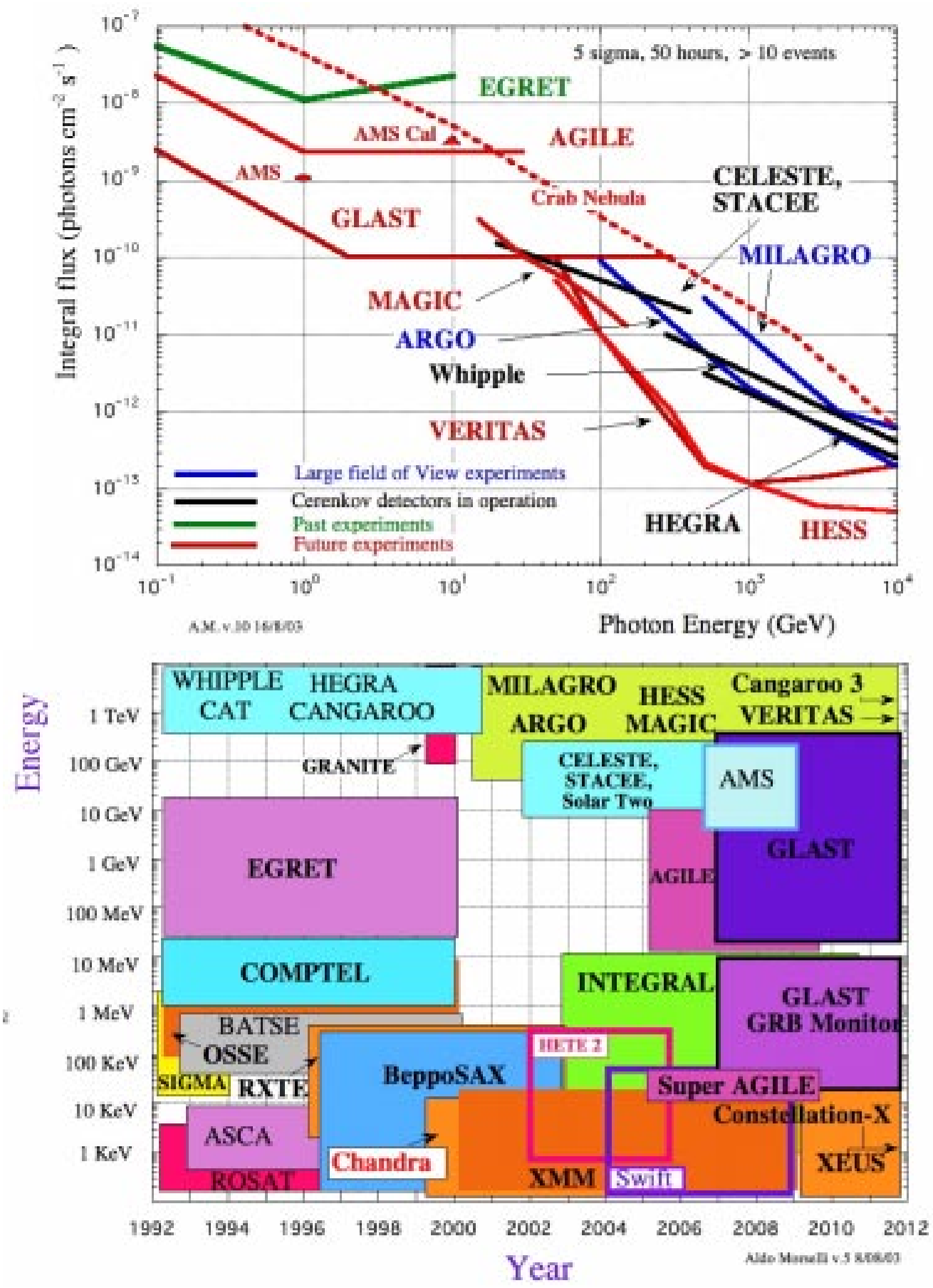

Figure 11: Sensitivity of present and future detectors in the gamma-ray astrophysics (top) and the timeline schedule versus the energy range covered by present and future detectors in $\mathrm{X}$ and gamma-ray astrophysics (bottom)

in other frequencies that will allow extensive multifrequency studies. 


\section{References}

[1] D.N. Spergel et al., Astrophys.J.Suppl. 148 (2003) 175 [astro-ph/0302209]

[2] L. Bergström, Rept. Prog. Phys. 63 (2000) 793

[3] A.Morselli, A. Lionetto, A. Cesarini, F. Fucito, P. Ullio, Nuclear Physics B 113B (2002) $213-220$

[4] A. Morselli, "Astroparticle and Gamma ray Physics in Space", Frascati Physics Series Vol.XXIV (2002), pp. 363-380, http://www.roma2.infn.it/infn/aldo/ISSS01.html

[5] http://www.roma2.infn.it/research/comm2/glast/

[6] A.Cesarini, F. Fucito, A. Lionetto, A.Morselli, Piero Ullio, submitted to Astroparticle Physics [astro-ph/0305075]

[7] L. J. Hall, J. Lykken and S. Weinberg, Phys. Rev. D 27 (1983) 2359.

[8] P. Gondolo, J. Edsjö, P. Ullio, L. Bergström, M. Schelke and E.A. Baltz, proceedings of idm2002, York, England, September 2002, astro-ph/0211238; http://www.physto.se/ ${ }^{2}$ edsjo/darksusy/.

[9] K. Hagiwara et al., Phys. Rev. D66 (2002) 010001.

[10] J. Edsjo, M. Schelke, P. Ullio and P. Gondolo, JCAP 0304 (2003) 001 [arXiv:hep-ph/0301106].

[11] Pythia program package, see T. Sjöstrand, Comp. Phys. Comm. 82 (1994) 74.

[12] A.Cesarini, F. Fucito, A. Lionetto, A.Morselli, Piero Ullio, in preparation

[13] J.F. Navarro, C.S. Frenk and S.D.M. White, Astrophys. J. 462 (1996) 563.

[14] S. Ghigna et al., Astrophys. J. 544 (2000) 616.

[15] E. Baltz and J. Edsjö, Phys. Rev. D 59 (1999) 023511

[16] M. Aguilar et al, Physics Reports, 366/6 (2002), 331

[17] P. Ullio 1999, astro-ph/9904086 and Frascati Physics Series Vol.XXIV (2002) 475 [http://www.roma2.infn.it/infn/aldo/ISSS01.html]

[18] G.Lamanna et al., Nuclear Physics B 113B (2002) 177

[19] P. Picozza and A. Morselli, Antimatter research in Space, Journal of Physics G: Nucl.Part.Phys. 29 (2003) 903-911 [astro-ph/0211286]

[20] S..Koushiappas et al. [astro-ph/0309464] 